\title{
Smoluchowski equation and the colloidal charge reversal
}

Cite as: J. Chem. Phys. 125, 054902 (2006); https://doi.org/10.1063/1.2222372

Submitted: 30 March 2006 . Accepted: 20 June 2006. Published Online: 01 August 2006

Alexandre Diehl, and Yan Levin

\section{ARTICLES YOU MAY BE INTERESTED IN}

Colloidal charge reversal: Dependence on the ionic size and the electrolyte concentration The Journal of Chemical Physics 129, 124506 (2008); https://doi.org/10.1063/1.2982163

Screening of a charged particle by multivalent counterions in salty water: Strong charge inversion

The Journal of Chemical Physics 113, 1110 (2000); https://doi.org/10.1063/1.481890

Charge inversion in electric double layers and effects of different sizes for counterions and coions

The Journal of Chemical Physics 108, 2940 (1998); https://doi.org/10.1063/1.475681

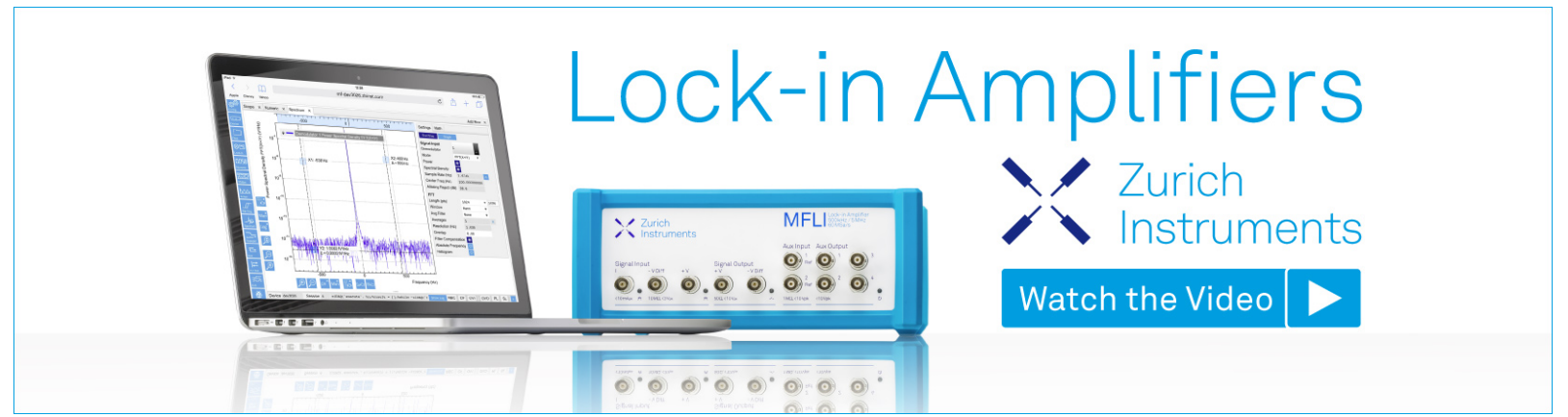

J. Chem. Phys. 125, 054902 (2006); https://doi.org/10.1063/1.2222372 


\title{
Smoluchowski equation and the colloidal charge reversal
}

\author{
Alexandre Diehl ${ }^{\mathrm{a})}$ \\ Departamento de Física, Universidade Federal do Ceará, Caixa Postal 6030, CEP 60455-760 Fortaleza, \\ Ceará, Brazil and Instituto de Física e Matemática, Universidade Federal de Pelotas, Caixa Postal \\ 354, CEP 96010-090, Pelotas, Rio Grande do Sul, Brazil \\ Yan Levin ${ }^{\text {b) }}$ \\ Instituto de Física, Universidade Federal do Rio Grande do Sul, Caixa Postal 15051, CEP 91501-970 \\ Porto Alegre, Rio Grande do Sul, Brazil
}

(Received 30 March 2006; accepted 20 June 2006; published online 1 August 2006)

\begin{abstract}
Smoluchowski equation and the Monte Carlo simulations are used to study the conditions leading to the reversal of the electrophoretic mobility. Zeta $(\zeta)$ potential is identified with the diffuse potential at the shear plane which, we argue, must be placed at least one ionic diameter away from the colloidal surface. For sufficiently strongly charged colloids, $\zeta$ potential changes sign as a function of the multivalent electrolyte concentration, resulting in a reversal of the electrophoretic mobility. This behavior occurs even for very small ions of $4 \AA$ diameter as long as the surface charge density of the colloidal particles is sufficiently large and the concentration of 1:1 electrolyte is sufficiently low. (C) 2006 American Institute of Physics. [DOI: 10.1063/1.2222372]
\end{abstract}

\section{INTRODUCTION}

Colloidal suspensions are ubiquitous. They are used in coatings, enhanced oil recovery, ceramics fabrication, biotechnology, and food industry, as well as many other applications. ${ }^{1}$ Nevertheless, our understanding of these systems is far from complete. To stabilize an aqueous suspension against flocculation, acidic groups are often synthesized on the surface of colloidal particles. When placed in water, these colloids acquire a net negative charge, repelling one another and preventing the irreversible flocculation from taking place. There are, however, a number of curious and often counterintuitive effects which occur in suspensions which in addition to $1: 1$ electrolyte also contain multivalent ions. ${ }^{2}$ For example, it has been observed that presence of multivalent counterions can lead to attraction between two like-charged colloidal particles. ${ }^{3-14}$ Similarly, it has been found that when transition metal ions such as $\mathrm{Mn}^{++}$and $\mathrm{Cd}^{++}$or polyamines such as spermine or spermidine are added to suspensions containing DNA strands, chains collapse forming toroidal bundles. ${ }^{15-20}$ Addition of multivalent ions to solutions containing actin filaments also results in interchain attraction and formation of rigid bundles. ${ }^{21,22}$

To probe the effective charge of colloidal particles and polyelectrolytes, a small electric field is applied to suspension and the drift velocity is measured. For sufficiently strongly charged colloids, it is observed that the electrophoretic mobility (the ratio between velocity and the applied electric field) can change sign, so that colloids will move in a direction opposite to the one expected based on their chemical charge. ${ }^{23-25}$ Reversal of the electrophoretic mobility is usually associated with the reversal of the effective colloidal charge., ${ }^{2,26}$ As the concentration of multivalent

\footnotetext{
${ }^{a)}$ Electronic mail: diehl@ fisica.ufc.br

${ }^{b)}$ Electronic mail: levin@if.ufrgs.br
}

counterions is increased, the strong electrostatic coupling between the counterions and colloids leads to the counterion condensation. The net charge of the resulting complexes can be very different from the bare charge, even differing in sign. A lot of theoretical work has been dedicated to find a way of calculating the effective charge of colloid-counterion complexes (see Refs. 2, 26, and 27 for review). One of the difficulties, however, seems to be that there is no unique way of defining the effective charge. Depending on the measurement performed, the effective charge of the complex will have a somewhat different value. ${ }^{28}$ The qualitative dependences of these effective charges on the parameters of suspension such as concentration of multivalent electrolyte, concentration of monovalent salt, colloidal bare charge, etc., are all very similar. $^{28}$

The most widely used definition of the effective charge is based on the Alexander prescription in which the "exact" electrostatic potential is asymptomatically matched to the solution of the linearized Poisson-Boltzmann equation. ${ }^{29-32}$ The prescription can be easily implemented for suspensions containing monovalent or multivalent counterions ${ }^{33}$ but no coions. It is also valid for aqueous suspensions containing small concentrations of 1:1 electrolyte but without multivalent counterions. Unfortunately, it appears to be impossible to extend this formalism to suspensions containing both multivalent counterions as well as any kind of coions. ${ }^{33,34}$ For such suspensions, one usually stipulates a geometrical region within which the counterions are taken to be associated with the colloidal particle. ${ }^{35}$ Although such theories can be quite useful for a qualitative understanding of the effective charge, the arbitrariness in the definition of the condensation sheath, in general, precludes a quantitative comparison with experiment.

In this work we will take a different approach. The electrophoretic mobility, in general, is a complicated nonlinear function of the electrokinetic $\zeta$ potential. ${ }^{1}$ For small $\zeta$ and 
large ionic strengths, however, the relationship between the two is linear and is given by a Smoluchowski equation. ${ }^{1,36}$ Since, close to the isoelectric point both $\zeta$ potential and the Debye length are small, the Smoluchowski equation is valid and the mobility measurements give us direct access to the value of the electrokinetic potential. Conversely, knowledge of the sign of $\zeta$ potential allows us to predict the direction of the electrophoretic drift inside a dilute colloidal suspension. The difficulty is that in general $\zeta$ potential is not known. The experimental measurements clearly show that the electrokinetic potential differs significantly from the bare surface potential. ${ }^{1}$ To use the Smoluchowski equation, the plane of shear must, therefore, be removed from the colloidal surface by some distance in order to account for the strongly bound counterions. The precise location of the shear plane will be determined by the combination of hydrodynamics and electrostatics. The recent molecular dynamics simulations of Joly et al. $^{37}$ indicate the basic correctness of this picture for normal wetting surfaces. The simulations also show that the shear planes are very close to the colloidal surface, removed from it by about one ionic monolayer. The precise location of the shear plane, however, varies slightly with the colloidal surface charge and the properties of the solvent. Following this observation, we will also identify the electrokinetic $\zeta$ potential with the mean electrostatic potential at the colloidal shear plane. ${ }^{36-39}$ The electrostatic potential will be calculated using Monte Carlo simulations. Since the precise position of the shear plane is not known to us exactly, our results can only be qualitative. However, we shall show that in the strong coupling limit, the counterion condensation results in an electrostatic potential which varies only weakly with distance. Thus, our expectation is that the precise location of the shear plane should not be very important, as long as it is about one ionic diameter away from the colloidal surface. This, however, should be checked by more detailed molecular dynamics simulations, including the explicit solvent.

\section{THE MODEL SYSTEM AND THE SIMULATION METHODOLOGY}

We consider a dilute aqueous colloidal suspension at room temperature containing a mixture of $1: 1$ and 3:1 electrolytes. The colloidal particles have surface charge $-Z q$ and their radius is fixed at $a_{c}=30 \AA$. The counterions and coions are modeled as hard spheres of radius $a=2 \AA$ with a point charge $z q$ at their center. The solvent is treated as a continuum of dielectric constant $\varepsilon$. The interaction potential between the ions (in mks units) is

$$
u_{i j}(r)=\left\{\begin{array}{cc}
\infty, & r<2 a \\
\frac{z_{i} z_{j} q^{2}}{4 \pi \varepsilon r}, & r \geqslant 2 a,
\end{array}\right.
$$

where $z_{i}$ is the valence of ion $i, r$ is the center-to-center distance, and $\varepsilon$ is the dielectric constant of water at room temperature, $\varepsilon=78 \varepsilon_{0}$. The interaction potential between colloid and ion $i$ is

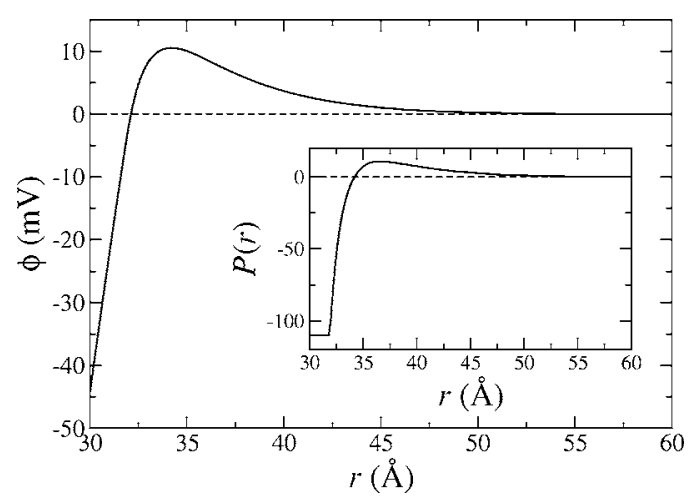

FIG. 1. Mean potential as a function of distance from colloidal center. The charge of colloid is $-110 q$ and the concentration of $3: 1$ electrolyte is $0.106 M$. Note the dramatic variation in the electrostatic potential over a distance of one ionic diameter $4 \AA$ from colloidal surface. The inset shows the integrated charge within a sphere of radius $r$ from colloidal center.

$$
u_{i}(r)=\left\{\begin{array}{cc}
\infty, & r<\left(a_{c}+a\right) \\
-\frac{Z z_{i} q^{2}}{4 \pi \varepsilon r}, & r \geqslant\left(a_{c}+a\right) .
\end{array}\right.
$$

The mean electrostatic potential at distance $r$ from the colloidal particle is

$$
\phi(r)=\int_{r}^{\infty} d r^{\prime} E\left(r^{\prime}\right)=\frac{q}{4 \pi \varepsilon} \int_{r}^{\infty} d r^{\prime} \frac{P\left(r^{\prime}\right)}{r^{\prime 2}},
$$

where $E(r)$ is the electric field and $P(r)$ is the integrated charge (in units of $q$ ) within a distance $r$ from the center of the colloidal particle. In the last equality of Eq. (3) Gauss's law was applied.

To calculate $P(r)$ we use a canonical Monte Carlo $(N V T)$ simulation. The electrostatic interactions are computed using the Ewald summation method with 518 Fourier-space wave vectors and a real-space damping parameter $\kappa=5$ (in units of simulation box size $L) .{ }^{40}$ Colloidal particle is located at the center of a cubic simulation box and is surrounded by the counterions and coions, the number of which satisfies the overall charge neutrality. The box length is fixed at $L$ $=120 \AA$, large enough to produce very small colloidal volume fractions, thus minimizing influence of periodicity on the ionic distribution inside the simulation box. This approach permits us to accurately model the ionic distribution in a very dilute bulk suspension, avoiding the boundary effects which are present if a canonical simulation is performed inside a hard Wigner-Seitz cell.

Two types of moves are utilized-ion transfer to a new random position and a small linear displacement. Acceptance ratios follow the standard Metropolis algorithm. The number of microions in each simulation is varied from approximately 50 up to 3000 particles, depending on the molar salt concentration. Typical runs involve $10^{7}$ Monte Carlo steps for equilibration and $10^{8}$ steps for production. Since the typical integrated charge rapidly decays to zero, see Fig. 1, the upper cutoff in Eq. (3) is taken to be $L / 2$.

In Fig. 1 we plot a characteristic mean electrostatic potential for a strongly charged colloid. The variation of potential is very rapid. It changes sign near the colloidal surface and reaches maximum at $r \approx a_{c}+2 a$. This suggests that a 
natural location of the shear plane is at about one ionic diameter from the colloidal surface. Traditionally the shear plane is placed at one ionic radius from the colloidal surface. ${ }^{41}$ This definition, however, does not take into account the thermal fluctuations and overlooks the strong variation of the mean electrostatic potential over a length of one ionic diameter, Fig. 1. The rapid growth of the electrostatic potential over a very short distance clearly shows that even ions which are not in a direct physical contact with the colloid are strongly bound to it by Coulomb force. The solvent induced shear stresses will, therefore, be applied not directly at the colloidal surface but at $r \approx a_{s} \equiv a_{c}+2 a$. This observation is also in agreement with the recent molecular dynamics simulation of electrophoretic motion of a small charged particle in a low salt suspension. ${ }^{42}$ The authors of this work measured the ion-colloid velocity cross-correlation function and found that the plateau region in its cross section extends beyond the surface of colloid by about one ionic diameter. This, again, shows that ions whose centers are removed from the colloidal surface by one ionic diameter still closely follow the colloidal motion. In view of these observations we define $\zeta \equiv \phi\left(a_{s}\right)$. We stress, however, that identification of the electrokinetic $\zeta$ potential with the mean electrostatic potential at the position of an effective shear plane is far from trivial. It can only be justified by appealing to the full molecular dynamics simulations. ${ }^{37,42}$ However, existence of a maximum in the mean electrostatic potential and the fact that $\phi(r)$ varies only weakly for $r \geqslant a_{c}+2 a$ make the precise location of the effective shear plane not very important as long as $a_{s} \geqslant a_{c}+2 a$.

To have a full understanding of the electrophoresis problem, a simultaneous solution of the Poisson and the NavierStokes equations is required. For aqueous solutions with only 1:1 electrolyte, ionic correlations can be ignored and the electrostatics can be treated at the mean-field PoissonBoltzmann level. Under these conditions O'Brien and White $^{43}$ where able to develop a computational scheme, which allowed them to numerically solve the system of Poisson-Boltzmann and the Navier-Stokes equations. In particular, they found that, in general, the electrophoretic mobility is a complicated function of $\zeta$ potential and the ionic strength. For small $\zeta$ and large ionic strengths, O'Brien and White recovered the Smoluchowski equation. For larger values of the electrokinetic potential, they found, however, that the electrophoretic mobility is no longer a monotonic function of $\zeta$. The nonmonotonicity of the electrophoretic mobility is a consequence of the polarization of the diffuse layer produced by the external electric field. In suspensions containing multivalent counterions in addition to monovalent salt, the behavior of the electrophoretic mobility can be expected to be even more complex. The key observation, however, is that the mobility remains linear in $\zeta$ and follows the Smoluchowski equation as long as $\zeta$ is small. This means that near the point of charge reversal $(\zeta \approx 0)$, the Smoluchowski equation can be applied without any restrictions. It is precisely the fact that the mobility is proportional to $\zeta$ potential [and not to the bare charge or the bare colloidal potential $\left.\phi\left(a_{c}\right)\right]$ that makes $\zeta$ potential relevant for the study of colloidal charge reversal. While the bare charge/potential

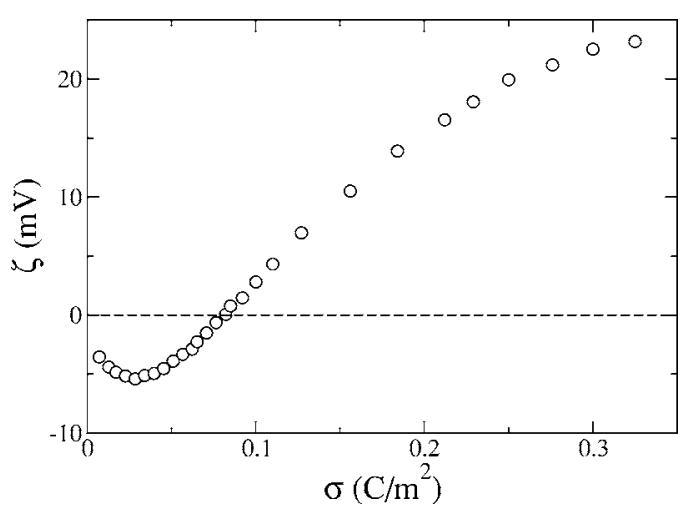

FIG. 2. Zeta potential as a function of colloidal surface charge density. The molar concentration of trivalent salt is $C_{+3}=0.1 \mathrm{M}$ and of monovalent salt is $C=0 M$.

can be very large, the effective charge and the $\zeta$ potential remain small near the point of reversal. Care, however, must be taken not to use the Smoluchowski equation outside the range of its validity. This, however, is never a problem in the present study, since we are only interested in exploring the conditions for the colloidal charge reversal, that is, when $\zeta$ $\approx 0$.

Recent theoretical calculations ${ }^{35}$ predict that the charge reversal with trivalent counterions of radius $a=2 \AA$ is possible, if and only if the magnitude of colloidal surface charge density, $\sigma=Z q / 4 \pi a_{c}^{2}$, is larger than the critical value $\sigma_{\mathrm{cr}}$ $=0.056 \mathrm{C} / \mathrm{m}^{2}$. This is a necessary but not a sufficient condition. The theory also predicts that even if $\sigma>\sigma_{\mathrm{cr}}$, overcharging will occur only if the monovalent salt concentration is below the critical threshold $C_{\mathrm{cr}}\left(Z, a_{c}\right)$. One of the goals of the present work is to test how much of these theoretical predictions apply to the reversal of the electrophoretic mobility.

\section{RESULTS AND DISCUSSION}

In Fig. 2 we present the $\zeta$ potential as a function of colloidal surface charge density for a suspension containing $3: 1$ electrolyte at concentration $C_{+3}=0.1 M$. For weakly charged colloidal particles, the increase (in modulus) of the surface charge density is accompanied by a uniform decline of $\zeta$ potential ( $\zeta$ accompanies the colloidal charge and becomes more negative). However, for sufficiently strongly charged colloids, the counterion condensation becomes important and $\zeta$ actually increase as a function of the bare colloidal charge, becoming positive for sufficiently strongly charged colloids. When this happens, the electrophoretic mobility of colloidal particles becomes reversed. It is interesting to note that the behavior of the $\zeta$ potential closely accompanies that of the effective charge. ${ }^{35}$ Of course, theories which do not explicitly take into account the interionic correlations fail to capture the reversal of the electrophoretic mobility for sufficiently strongly charged colloids. This is specifically the case of the Poisson-Boltzmann (PB) theory, which predicts that the effective colloidal charge will saturate without ever becoming reversed. ${ }^{29}$ For comparison, in Fig. 3 we show the dependence of $\zeta$ potential on the colloidal surface charge density when suspension contains only 1:1 electrolyte, and 


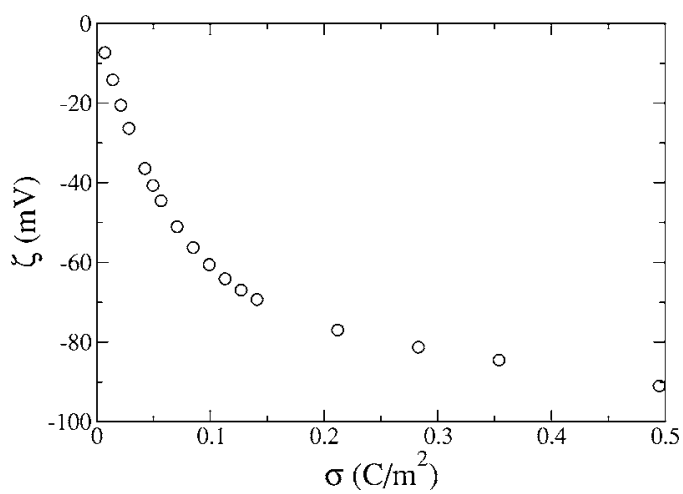

FIG. 3. Zeta potential as a function of colloidal surface charge density when there is only $1: 1$ electrolyte at $C=0.0258 \mathrm{M}$.

the counterion correlations are not very important. In this case $\zeta$ remains always negative and there is no reversal of the electrophoretic mobility at any value of the colloidal surface charge density. Again, the behavior of the $\zeta$ potential is very similar to that of the effective colloidal charge, showing the characteristic Poisson-Boltzmann saturation at larger values of $\sigma$.

We next consider the dependence of $\zeta$ potential on the concentration of trivalent counterions, Fig. 4. One can see that there is a minimum colloidal surface charge density below which charge reversal does not take place however large the concentration of trivalent ions inside the suspension is. The critical surface charge is found to be $\sigma_{\mathrm{cr}} \approx 0.050 \mathrm{C} / \mathrm{m}^{2}$, the value which is in good agreement with the theoretical prediction $^{35}$ of $0.056 \mathrm{C} / \mathrm{m}^{2}$.

When 1:1 electrolyte is added to colloidal suspension, the particles of which are already overcharged by a 3:1 electrolyte, $\zeta$ potential decreases, see Fig. 5 . We find a very good linear fit of $\zeta$ with the concentration of 1:1 electrolyte. Above the critical threshold, charge reversal disappears. This behavior is contrary to the predictions of the calculations of Nguyen et $a{ }^{44}$ who argued that stronger screening of the electrostatic self-energy of the overcharged polyioncounterion complexes should result in a "giant" charge reversal. On the other hand, the theory of Pianegonda et al. ${ }^{35}$ finds that for large salt concentrations multivalent ions prefer

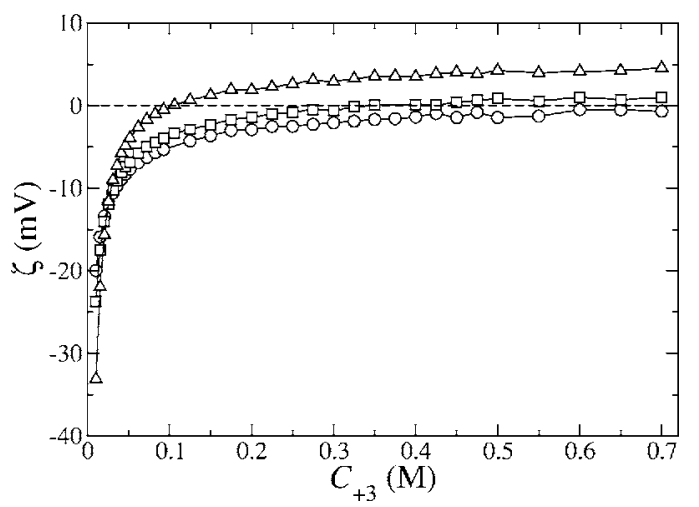

FIG. 4. Zeta potential as a function of the molar concentration of trivalent ions. Circles, squares, and triangles represent the simulation data for $Z=30$, 40 , and 58, respectively, corresponding to surface charge densities $\sigma$ $\approx 0.042,0.057$, and $0.082 \mathrm{C} / \mathrm{m}^{2}$, respectively. The solid lines are merely guides to the eye.

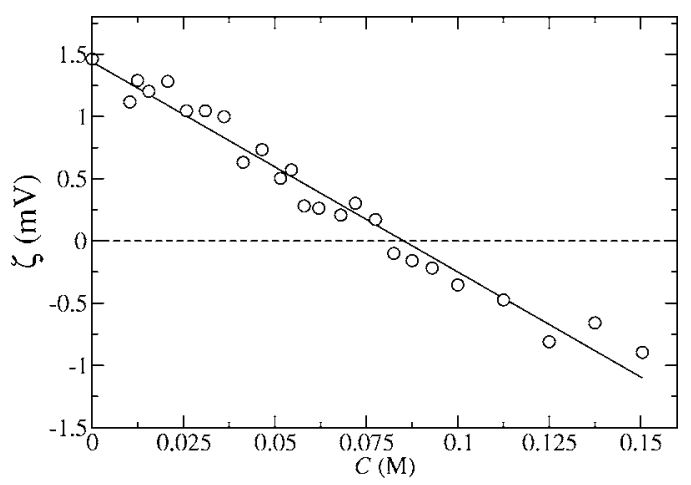

FIG. 5. Zeta potential as a function of the molar concentration of monovalent salt for a colloidal particle of $Z=65$ and multivalent salt concentration $C_{+3}=0.106 M$. Solid line represents a fit to the simulation data.

to be solvated in the bulk electrolyte where they gain favorable correlational energy from the interactions with oppositely charged coions depleted from the colloidal surface. This finding is consistent with our Monte Carlo (MC) simulations.

In practice, dissociation of water always provides a small concentration of 1:1 electrolyte; however, as can be seen from Fig. 5, this has a negligible effect on the reversal of the electrophoretic mobility. Finally, we mention that the theory of Pianegonda et al. ${ }^{35}$ predicts that very small concentrations of 1:1 electrolyte should actually enhance slightly the degree of charge reversal. If this conclusion is also applicable to the reversal of the electrophoretic mobility is not clear, since strong fluctuations prevent us from clearly exploring this very dilute regime of 1:1 electrolyte.

\section{CONCLUSIONS}

Canonical Monte Carlo simulations were used to study mean electrostatic potential of suspensions containing a mixture of 3:1 and 1:1 electrolytes. It was argued that due to strong Coulomb attraction between colloids and condensed counterions, the shear plane must be moved to $r \approx a_{s} \equiv a_{c}$ $+2 a$ and the electrokinetic $\zeta$ potential can be identified with the diffuse electrostatic potential at $r=a_{s}$. For strongly charged colloids, this location is where the mean electrostatic potential is near its maximum and, therefore, is quite insensitive to small perturbations in the precise position of the hydrodynamic slip plane. In this respect our philosophy is very close to the one adopted by Bjerrum in his original study of ionic clusters. ${ }^{45,46}$ On the other hand, locating the shear plane at the traditional position $r \approx a_{c}+a$ leads to $\zeta$ potential strongly dependent on any small perturbation, see Fig. 1, which is unacceptable from a theoretical point of view. It would also lead to an incorrect conclusion that there is no reversal of the electrophoretic mobility in cases when there is a clear reversal of the colloidal charge, as seen from the behavior of $P(r)$, Fig. 1. Without solving the complete hydrodynamic problem or performing a full atomistic simulation, however, our results can only be considered qualitative.

The simulations show that there is a critical charge density below which the reversal of the electrophoretic mobility is impossible however large the concentration of the multi- 
valent electrolyte in suspension is. Addition of 1:1 electrolyte leads to diminished counterion-colloid association and, thus, hinders the charge reversal. Both of these results are in agreement with the resent theoretical predictions. ${ }^{35}$

Reversal of the electrophoretic mobility is driven by the electrostatic correlations. ${ }^{2,26}$ Strong positional correlations between the counterions enhance the counterion condensation over the predictions of mean-field theories and, for sufficiently strongly charged particles, can lead to colloidal charge reversal. ${ }^{4-49}$ On the other hand, attraction between the counterions and coion (depleted from the colloidal surface) favors solvation of the multivalent ions in the bulk of suspension. The stronger of the two effects will determine if the colloidal charge reversal takes place. Since salt lowers the bulk chemical potential of multivalent ions, its addition (at moderate concentrations) makes the counterion condensation less favorable and hinders the charge reversal. ${ }^{35,50}$ Similarly diminishing the ionic diameter leads to stronger counterion-coion interactions, preventing the charge segregation from taking place near the colloidal surface. A theoretical argument ${ }^{51}$ suggests that the critical surface charge density diverges when the radius of counterions goes to zero as $\sigma_{\mathrm{cr}} \sim 1 / a^{2}$.

\section{ACKNOWLEDGMENT}

This work was supported by the Brazilian agency CNPq.

${ }^{1}$ W. B. Russel, D. A. Saville, and W. R. Schowalter, in Colloidal Dispersions, edited by G. K. Batchelor (Cambridge University Press, Cambridge, 1989).

${ }^{2}$ Y. Levin, Rep. Prog. Phys. 65, 1577 (2002).

${ }^{3}$ G. N. Patey, J. Chem. Phys. 72, 5763 (1980).

${ }^{4}$ R. Kjellander and S. Marcelja, Chem. Phys. Lett. 112, 49 (1984).

${ }^{5}$ L. Guldbrand, B. Jonsson, H. Wennerstrom, and P. Linse, J. Chem. Phys. 80, 2221 (1984).

${ }^{6}$ M. J. Stevens and M. O. Robbins, Europhys. Lett. 12, 81 (1990).

${ }^{7}$ J. M. Crocker and D. G. Grier, Phys. Rev. Lett. 73, 352 (1994).

${ }^{8}$ N. Grønbech-Jensen, R. J. Mashl, R. F. Bruinsma, and W. M. Gelbart, Phys. Rev. Lett. 78, 2477 (1997)

${ }^{9}$ E. Allahyarov, I. D'Amico, and H. Lowen, Phys. Rev. Lett. 81, 1334 (1998)

${ }^{10}$ Y. Levin, Physica A 265, 432 (1999).

${ }^{11}$ A. W. C. Lau, P. Pincus, D. Levine, and H. A. Fertig, Phys. Rev. E 63 , 051604 (2001).

${ }^{12}$ D. G. Grier, J. Phys.: Condens. Matter 12, A85 (2000).

${ }^{13}$ V. Lobaskin, A. Lyubartsev, and P. Linse, Phys. Rev. E 63, 020401 (2001).

${ }^{14}$ A. Naji and R. R. Netz, Eur. Phys. J. E 13, 43 (2004).

${ }^{15}$ A. A. Kornyshev and S. Leikin, Phys. Rev. Lett. 82, 4138 (1999).
${ }^{16}$ I. Rouzina and V. Bloomfield, J. Chem. Phys. 100, 9977 (1996).

${ }^{17}$ J. J. Arenzon, J. F. Stilck, and Y. Levin, Eur. Phys. J. B 12, 79 (1999).

${ }^{18}$ F. J. Solis and M. O. de la Cruz, Phys. Rev. E 60, 4496 (1999).

${ }^{19}$ W. M. Gelbart, R. F. Bruinsma, P. A. Pincus, and V. A. Parsegian, Phys. Today 53(9), 38 (2000).

${ }^{20}$ F. J. Solis and M. O. de la Cruz, Phys. Today 54(1), 71 (2001).

${ }^{21}$ J. X. Tang and P. A. Janmey, J. Biol. Chem. 271, 8556 (1996).

${ }^{22}$ T. E. Angelini, H. Liang, W. Wrigglers, and G. C. L. Wong, Proc. Natl. Acad. Sci. U.S.A. 100, 8634 (2003).

${ }^{23}$ M. Quesada-Pérez, J. Callejas-Fernández, and R. Hidalgo-Álvarez, Adv. Colloid Interface Sci. 95, 295 (2002).

${ }^{24}$ A. Martin-Molina, M. Quesada-Perez, F. Galisteo-Gonzalez, and R. Hidalgo-Alvarez, J. Phys.: Condens. Matter 15, S3475 (2003).

${ }^{25}$ A. Fernandez-Nieves, A. Fernandez-Barbero, F. J. de las Nieves, and B. Vincent, J. Chem. Phys. 123, 054905 (2005).

${ }^{26}$ A. Y. Grosberg, T. T. Nguyen, and B. I. Shklovskii, Rev. Mod. Phys. 74, 329 (2002).

${ }^{27}$ L. Belloni, Colloids Surf., A 140, 227 (1998).

${ }^{28}$ P. Wette, H. J. Schope, and T. Palberg, J. Chem. Phys. 116, 10981 (2002).

${ }^{29}$ S. Alexander, P. M. Chaikin, P. Grant, G. J. Morales, P. Pincus, and D. Hone, J. Chem. Phys. 80, 5776 (1984).

${ }^{30}$ E. Trizac, L. Bocquet, and M. Aubouy, Phys. Rev. Lett. 89, 248301 (2002).

${ }^{31}$ L. Bocquet, E. Trizac, and M. Aubouy, J. Chem. Phys. 117, 8138 (2002).

${ }^{32}$ E. Trizac and Y. Levin, Phys. Rev. E 69, 031403 (2004).

${ }^{33}$ A. Diehl and Y. Levin, J. Phys.: Condens. Matter 17, S3309 (2005).

${ }^{34}$ L. Samaj, J. Stat. Phys. 119, 459 (2005).

${ }^{35}$ S. Pianegonda, M. C. Barbosa, and Y. Levin, Europhys. Lett. 71, 831 (2005).

${ }^{36}$ R. J. Hunter, Zeta Potential in Colloidal Science: Principles and Applications (Academic, London, 1981).

${ }^{37}$ L. Joly, C. Ybert, E. Trizac, and L. Bocquet, Phys. Rev. Lett. 95, 257805 (2004).

${ }^{38}$ Y. W. Kim and R. R. Netz, Europhys. Lett. 72, 837 (2005).

${ }^{39}$ J. Lyklema, Fundamentals of Interface and Colloid Science (Academic, New York, 1995).

${ }^{40}$ M. P. Allen and D. J. Tildesley, Computer Simulations of Liquids (Oxford University Press, Oxford, 1987).

${ }^{41}$ M. Quesada-Pérez, A. Martín-Molina, and R. Hidalgo-Alvarez, Langmuir 21, 9231 (2005).

${ }^{42}$ V. Lobaskin, B. Dünweg, M. Medebach, T. Palberg, and C. Holm, e-print cond-mat/0601588..

${ }^{43}$ R. W. O'Brien and L. R. White, J. Chem. Soc., Faraday Trans. 2 74, 1607 (1978).

${ }^{44}$ T. T. Nguyen, A. Y. Grosberg, and B. I. Shklovskii, Phys. Rev. Lett. 85, $1568(2000)$

${ }^{45}$ N. Bjerrum, K. Dan. Vidensk. Selsk. Mat. Fys. Medd. 7, 1 (1926).

${ }^{46}$ Y. Levin and M. E. Fisher, Physica A 225, 164 (1996).

${ }^{47}$ G. M. Torrie and J. P. Valleau, J. Chem. Phys. 73, 5807 (1980).

${ }^{48}$ M. Lozada-Cassou, R. Saavedra-Barrera, and D. Henderson, J. Chem. Phys. 77, 5150 (1982).

${ }^{49}$ M. Deserno, F. Jimenez-Angeles, C. Holm, and M. Lozada-Cassou, J. Phys. Chem. 105, 10983 (2001).

${ }^{50}$ F. J. Solis and M. O. de la Cruz, Eur. Phys. J. E 4, 143 (2001).

${ }^{51}$ Y. Levin, J. Phys.: Condens. Matter 16, S2149 (2004). 